\title{
Need for a Different Strategy for Breast Cancer Screening in India: Reply to Letter
}

\author{
Gaurav Agarwal · Pooja Ramakant
}

Published online: 4 March 2010

(C) Société Internationale de Chirurgie 2010

We thank Drs. Gadgil and Roy for their letter to editor [1] about our article and for supporting our view for the need for a different strategy for breast cancer screening in India [2]. Drs Gadgil and Roy have reiterated the facts that epidemiology and clinical presentation of breast cancer in India continue to be starkly different from those in the industrialized world. They have also re-emphasized the fact stated by us that the rise in the incidence of breast cancer has been documented more in the urban areas than in the rural areas and that cancer of the uterine cervix remains the most common cancer afflicting rural women in India. In metropolitan cities breast cancer has overtaken other cancers as the most common cancer in women. The meager public health funds in India, which are directed mostly to treating patients with infectious and cardiovascular diseases, cannot support "expensive" mass mammography screening. With the relatively low incidence of breast cancer, the efficacy and cost effectiveness of mammographic screening is highly questionable in India. In the study undertaken by the authors at their community hospital, they report no detection of invasive breast cancers in patients screened over 10 years [1]. They further provide estimates of the high costs of screening a population of 95,000 in their community, and raise the concern of falsepositive mammographies and the anxiety and unnecessary concern those can create [1].

G. Agarwal $(\bowtie) \cdot$ P. Ramakant

Department of Endocrine and Breast Surgery, Sanjay Gandhi

Postgraduate Institute of Medical Sciences, Raebareli Road,

Lucknow 226014, India

e-mail: gaurav@sgpgi.ac.in
At this juncture, given that a majority of breast cancers are still detected at a locally advanced stage in India, efforts must be concentrated toward (1) improving health awareness in general, and breast awareness in particular; (2) encouraging breast self-examination as a method of improving breast awareness, but not as a method of screening or early detection; and (3) annual breast examination by a physician or other health-care worker in the community [3].

Also, given the fact that many patients who are aware of a breast lump postpone or shun medical care due to ignorance or fear of expensive and painful treatment, efforts must be made to educate the general population that breast cancer is curable and to make appropriate multimodality treatment available to the poorest of the poor either free of cost or at highly subsidized costs. At the same time, for urban women who are increasingly embracing the westernized lifestyle and are in general believed to be at a much higher risk of developing breast cancer, appropriate breast awareness measures as well as screening measures like an annual breast examination by a physician, opportunistic mammographic screening, and routine mammographic screening for individuals specifically at high risk must also be ensured [3].

\section{References}

1. Gadgil A, Roy N (2010) Need for a different screening strategy for breast cancer in India: letter to the Editor. World J Surg 34

2. Agarwal G, Ramakant P, Sánchez Forgach ER et al (2009) Breast cancer care in developing countries. World J Surg 33(10):20692076

3. Agarwal G, Ramakant P (2008) Breast cancer care in India: current situation and challenges for the future. Breast Care 3:21-27 